\title{
$A$-differentiability and $A$-analyticity *
}

\author{
P. M. Gadea and J. Muñoz Masqué
}

\begin{abstract}
Let $A$ be a finite-dimensional commutative algebra over $\mathbb{R}$ and let $C_{A}^{r}(U), C^{\omega}(U, A)$ and $\mathcal{O}_{A}(U)$ be the ring of $A$-differentiable functions of class $C^{r}, 0 \leq r \leq \infty$, the ring of real analytic mappings with values in $A$ and the ring of $A$-analytic functions, respectively, defined on an open subset $U$ of $A^{n}$. We prove two basic results concerning $A$-differentiability and $A$-analyticity: $\left.\left.1^{\text {st }}\right) \mathcal{O}_{A}(U)=C_{A}^{\infty}(U) \bigcap C^{\omega}(U, A), 2^{\text {nd }}\right) \mathcal{O}_{A}(U)=C_{A}^{\infty}(U)$ if and only if $A$ is defined over $\mathbb{C}$.
\end{abstract}

\section{Preliminaries and statement of the main re- sults}

Let $A$ be a finite-dimensional commutative $\mathbb{R}$-algebra. Let us denote by $G(A)$ the group of units of $A$. Clearly, $G(A)$ is an open dense subset of $A$ and hence it is endowed with a canonical structure of Lie group. Let $U$ be an open subset of $A$. A function $f: U \longrightarrow A$ is said to be $A$-differentiable if for every $a \in U$ there exists the limit $f^{\prime}(a)=\lim _{x \rightarrow a}(f(x)-f(a)) /(x-a), x-a \in G(A)$. We say that $f$ is $A$-differentiable of class $C^{r}$ and set $f \in C_{A}^{r}(U)$ if for every $a \in$ $U, f^{\prime}(a), f^{\prime \prime}(a), \ldots, f^{(r)}(a)$ exist and they are continuous. For further properties on $A$-differentiability we send to $[2,3,4]$.

As is well-known, if $V$ is a finite-dimensional $\mathbb{R}$-vector space, for each open subset $U \subset V$ we have a canonical isomorphism $\zeta_{x}: V \longrightarrow T_{x}(U)$ given by $\zeta_{x}(v)(f)=\lim _{t \rightarrow 0} \frac{1}{t}(f(x+t v)-f(x))$ (directional derivative). In the particular case $V=A$ we can define a family of endomorphisms on each tangent space $h_{a}: T_{x}(U) \longrightarrow T_{x}(U), a \in A, h_{a}(X)=\zeta_{x}\left(a \cdot \zeta_{x}^{-1}(X)\right)$. Then, it is proved that the definition of the analyst coincides with that of the differential geometer (this is not the case for non-commutative algebras, see [2]); i.e., a function $f: U \longrightarrow A$ of class $C^{\infty}$ is A-differentiable if and only if for every $a \in A, x \in U$, the mapping $f_{*}: T_{x}(U) \longrightarrow T_{x}(U)$ commutes with $h_{a}$.

The product in $A$ induces an $\mathbb{R}$-bilinear mapping $\mu: A \times A \longrightarrow A$. Let $e_{0}=1, e_{1}, \ldots, e_{m-1}$ be a basis of $A$ as an $\mathbb{R}$-vector space. Then, $\mu\left(e_{i}, e_{j}\right)=$ $\sum_{k=0}^{m-1} \mu_{i j}^{k} e_{k}, 0 \leq i, j \leq m-1$. Each function $f: U \longrightarrow A$ can be written as $f(x)=\sum_{i=0}^{m-1} f_{i}(x) e_{i}$ and $f$ is $A$-differentiable if and only if $\partial f / \partial x_{j}=$

*A.M.S. Subject Classification Number: Primary 30G35; Secondary 26E05, 26E10,16P10. Supported by DGICYT (Spain) grant n. PB89-0004. 
$\left(\partial f / \partial x_{0}\right) e_{j}$ for every $j=1, \ldots, m-1$; or more explicitly, $\partial f_{i} / \partial x_{j}=\sum_{h=0}^{m-1} \mu_{h j}^{i}\left(\partial f_{h} / \partial x_{0}\right), i=$ $0, \ldots, m-1 ; j=1, \ldots, m-1$ (Cauchy-Riemann equations). It follows that $C_{A}^{r}(U)$ is a closed $A$-subalgebra of $C^{r}(U, A)$ with respect to its natural topology of Fréchet algebra.

The above definitions and results can also be extended to several variables. Let $U$ be an open subset of $A^{n}$. A function $F: U \longrightarrow A$ is said to be $A$ differentiable if for every $x=\left(x_{1}, \ldots, x_{n}\right) \in U$ and every $i=1, \ldots, n$ the function

$$
y \mapsto F\left(x_{1}, \ldots, x_{i-1}, y, x_{i+1}, \ldots, x_{n}\right),
$$

defined on a neighbourhood of $x_{i}$, is $A$-differentiable. Set $x_{i}=\sum_{j=0}^{m-1} x_{i j} e_{j}, i=$ $1, \ldots, n$. We obtain: $F$ is A-differentiable if and only if $\partial F / \partial x_{i j}=\left(\partial F / \partial x_{i 0}\right) e_{j}, i=$ $1, \ldots, n ; j=1, \ldots, m-1$. Finally, $F$ is said to be $A$-analytic and then we set $F \in \mathcal{O}_{A}(U)$ if for every $x^{0}=\left(x_{1}^{0}, \ldots, x_{n}^{0}\right) \in U$ there exists a series $\sum_{\alpha \in \mathbb{N}^{n}} a_{\alpha}\left(x_{1}-\right.$ $\left.x_{1}^{0}\right)^{\alpha_{1}} \cdots\left(x_{n}-x_{n}^{0}\right)^{\alpha_{n}}$ absolutely convergent on $\left|x_{i}\right| \leq r_{i}, i=1, \ldots, n$, such that for every $x=\left(x_{1}, \ldots, x_{n}\right) \in U,\left|x_{i}-x_{i}^{0}\right| \leq r_{i}, i=1, \ldots, n$, we have

$$
F(x)=\sum_{\alpha \in \mathbb{N}^{n}} a_{\alpha}\left(x_{1}-x_{1}^{0}\right)^{\alpha_{1}} \cdots\left(x_{n}-x_{n}^{0}\right)^{\alpha_{n}} .
$$

Our basic results are the following two theorems:

Theorem I. The ring of A-analytic functions on an open set $U \subset A^{n}$, is the intersection of the ring of A-differentiable functions of class $C^{\infty}$ on $U$, and the ring of real analytic A-valued mappings defined on $U$; i.e., $\mathcal{O}_{A}(U)=$ $C_{A}^{\infty}(U) \cap C^{\omega}(U, A)$.

Theorem II. $\mathcal{O}_{A}(U)=C_{A}^{\infty}(U)$ if and only if $A$ is defined over $\mathbb{C}$.

The latter theorem can also be restated as follows:

Corollary. $\mathcal{O}_{A}(U)=C_{A}^{\infty}(U)$ if and only if $G(A)$ is connected.

\section{Proof of Theorem I}

Lemma 1. Let $F: U \longrightarrow A, U \subset A^{n}$, be an A-differentiable function of class $C^{r}$. For every system of indices $i_{1}, \ldots, i_{k}, j_{1}, \ldots, j_{k}$, such that $1 \leq i_{h} \leq n$, $0 \leq j_{h} \leq m-1,1 \leq h \leq k \leq r$, we have

$$
\frac{\partial^{k} F}{\partial x_{i_{1} j_{1}} \ldots \partial x_{i_{k} j_{k}}}=\frac{\partial^{k} F}{\partial x_{i_{1}} 0 \cdots \partial x_{i_{k}} 0} e_{j_{1}} \cdots e_{j_{k}} .
$$

Proof. We proceed by recurrence on $k$. For $k=1$, the above formula is nothing but the Cauchy-Riemann equations for $F$ (cf. $\$ 1$ ), and by virtue of the recurrence hypothesis for every $k>1$, we obtain

$$
\partial^{k} F / \partial x_{i_{1} j_{1}} \ldots \partial x_{i_{k} j_{k}}=\partial / \partial x_{i_{1} j_{1}}\left(\partial^{k-1} F / \partial x_{i_{2} j_{2}} \ldots \partial x_{i_{k} j_{k}}\right)
$$


$=\partial / \partial x_{i_{1} j_{1}}\left(\left(\partial^{k-1} F / \partial x_{i_{2} 0} \ldots \partial x_{i_{k} 0}\right) e_{j_{2}} \cdots e_{j_{k}}\right)=\left(\partial^{k} F / \partial x_{i_{1} 0} \ldots \partial x_{i_{k} 0}\right) e_{j_{1}} e_{j_{2}} \cdots e_{j_{k}}$.

Proof of Theorem I. First we prove $\mathcal{O}_{A}(U) \subset C_{A}^{\infty}(U) \cap C^{\omega}(U, A)$. If $F: U \longrightarrow A$ is $A$-analytic, given $x^{0}=\left(x_{1}^{0}, \ldots, x_{n}^{0}\right) \in U$, there exists a series $\sum_{\alpha} a_{\alpha} x_{1}^{\alpha_{1}} \cdots x_{n}^{\alpha_{n}}$ converging absolutely on $\left|x_{i}\right| \leq r_{i}, 1 \leq i \leq n$, such that for every $x=\left(x_{1}, \ldots, x_{n}\right) \in$ $U,\left|x_{i}-x_{i}^{0}\right| \leq r_{i}, 1 \leq i \leq n$, we have $F(x)=\sum_{\alpha \in \mathbb{N}^{n}} a_{\alpha}\left(x_{1}-x_{1}^{0}\right)^{\alpha_{1}} \cdots\left(x_{n}-\right.$ $\left.x_{n}^{0}\right)^{\alpha_{n}}$. With the above notations, i.e., $x_{i}=\sum_{j=0}^{m-1} x_{i j} e_{j}, 1 \leq i \leq n$, it is not difficult to see that $F(x)$ can be expressed as an absolutely convergent series of the real variables $x_{i j}-x_{i j}\left(x^{0}\right)$ taking values in $A$. Therefore, $F \in C^{\omega}(U, A)$. Moreover, from the standard properties of power series it follows that we can calculate $\partial F / \partial x_{i j}$ simply taking derivatives term by term. As $\left(\partial / \partial x_{i j}\right)\left(x_{h}-x_{h}^{0}\right)^{k}=k\left(x_{h}-x_{h}^{0}\right)^{k-1} \delta_{h i} e_{j}$, we have:

$$
\frac{\partial F}{\partial x_{i j}}=\sum_{\alpha \in \mathbb{N}^{n}} a_{\alpha} \alpha_{i}\left(x_{1}-x_{1}^{0}\right)^{\alpha_{1}} \cdots\left(x_{i}-x_{i}^{0}\right)^{\alpha_{i}-1} \cdots\left(x_{n}-x_{n}^{0}\right)^{\alpha_{n}} \cdot e_{j}=\frac{\partial F}{\partial x_{i 0}} e_{j} .
$$

Thus, $F$ is $A$-differentiable.

Next we shall give two proofs of the second part of Theorem I.

$1^{s t}$ ) Assume $F: U \longrightarrow A$ is a function of class $\mathcal{C}^{\omega}$. Then, its components $F=\sum_{k=0}^{m-1} F_{k} e_{k}$ can be expanded in power series on a neighbourhood of $x^{0} \in U$ :

$F_{k}(x)=\sum_{\left|\beta_{1}\right|, \ldots,\left|\beta_{n}\right|=0}^{\infty} \frac{1}{\beta_{1} ! \cdots \beta_{n} !} \frac{\partial^{\left|\beta_{1}\right|+\ldots+\left|\beta_{n}\right|}}{\partial x_{1}^{\beta_{1}} \cdots \partial x_{n}^{\beta_{n}}}\left(x^{0}\right)\left(x_{1}-x_{1}\left(x^{0}\right)\right)^{\beta_{1}} \cdots\left(x_{n}-x_{n}\left(x^{0}\right)\right)^{\beta_{n}}$,

where we have set:

$$
\begin{gathered}
\beta_{i}=\left(\beta_{i 0}, \ldots, \beta_{i, m-1}\right), i=1, \ldots, n, \quad \partial x_{i}^{\beta_{i}}=\partial x_{i 0}^{\beta_{i 0}} \cdots \partial x_{i, m-1}^{\beta_{i, m-1}}, \\
\left(x_{i}-x_{i}\left(x^{0}\right)\right)^{\beta_{i}}=\left(x_{i 0}-x_{i 0}\left(x^{0}\right)\right)^{\beta_{i 0}} \cdots\left(x_{i, m-1}-x_{i, m-1}\left(x^{0}\right)\right)^{\beta_{i, m-1}}, \\
\left|\beta_{i}\right|=\beta_{i 0}+\ldots+\beta_{i, m-1}, \quad \beta_{i} !=\beta_{i 0} ! \cdots \beta_{i, m-1} !
\end{gathered}
$$

Furthermore, we set:

$$
\begin{gathered}
\beta=\left(\beta_{1}, \ldots, \beta_{n}\right), \quad \beta !=\beta_{1} ! \cdots \beta_{n} !, \quad\left(x-x\left(x^{0}\right)\right)^{\beta}=\prod_{i=1}^{n}\left(x_{i}-x_{i}\left(x^{0}\right)\right)^{\beta_{i}}, \\
\partial^{|\beta|} F_{k} / \partial x^{\beta}=\partial^{\left|\beta_{1}\right|+\ldots+\left|\beta_{n}\right|} F_{k} / \partial x_{1}^{\beta_{1}} \cdots \partial x_{n}^{\beta_{n}} .
\end{gathered}
$$

Hence,

$$
F(x)=\sum_{k=0}^{m-1} \sum_{|\beta|=0}^{\infty} \frac{1}{\beta !}\left(\partial^{|\beta|} F_{k} / \partial x^{\beta}\right)\left(x^{0}\right)\left(x-x\left(x^{0}\right)\right)^{\beta} e_{k},
$$

and by virtue of Lemma 1 ,

$$
\left(\partial^{|\beta|} F / \partial x^{\beta}\right)\left(x^{0}\right)=\sum_{k=0}^{m-1}\left(\partial^{|\beta|} F_{k} / \partial x^{\beta}\right)\left(x^{0}\right) e_{k}
$$




$$
=\left(\partial^{|\beta|} F / \partial x_{10}^{\left|\beta_{1}\right|} \cdots \partial x_{n 0}^{\left|\beta_{n}\right|}\right)\left(x^{0}\right) e_{0}^{\beta_{10}} \cdots e_{m-1}^{\beta_{1, m-1}} \cdots e_{0}^{\beta_{n 0}} \cdots e_{m-1}^{\beta_{n, m-1}} .
$$

Hence,

$$
\begin{aligned}
& F(x)=\sum_{|\beta|=0}^{\infty} \frac{1}{\beta !} \frac{\partial^{|\beta|} F}{\partial x_{10}^{\left|\beta_{1}\right|} \cdots \partial x_{n 0}^{\left|\beta_{n}\right|}}\left(x^{0}\right)\left(x_{10}-x_{10}\left(x^{0}\right)\right)^{\beta_{10}} \cdots\left(x_{1, m-1}-x_{1, m-1}\left(x^{0}\right)\right)^{\beta_{1, m-1}} \\
& e_{0}^{\beta_{10}} \cdots e_{m-1}^{\beta_{1, m-1}} \cdots\left(x_{n 0}-x_{n 0}\left(x^{0}\right)\right)^{\beta_{n 0}} \cdots\left(x_{n, m-1}-x_{n, m-1}\left(x^{0}\right)\right)^{\beta_{n, m-1}} e_{0}^{\beta_{n 0}} \cdots e_{m-1}^{\beta_{n, m-1}} \\
& =\frac{1}{m !^{n}} \sum_{|\beta|=0}^{\infty} \frac{1}{\beta !} \frac{\partial^{|\beta|} F}{\partial x_{10}^{\left|\beta_{1}\right|} \cdots \partial x_{n 0}^{\left|\beta_{n}\right|}}\left(x^{0}\right) \prod_{i=1}^{n}\left(x_{i}-x_{i}^{0}\right)^{\left|\beta_{i}\right|},
\end{aligned}
$$

as follows by applying to $\left(x_{i}-x_{i}^{0}\right)^{\left|\beta_{i}\right|}=\left(\sum_{k=0}^{m-1}\left(x_{i k}-x_{i k}\left(x^{0}\right)\right) e_{k}\right)^{\left|\beta_{i}\right|}$ the Leibniz's formula.

By setting

$\alpha_{i}=\left|\beta_{i}\right|, i=1, \ldots, n, \alpha=\left(\alpha_{1}, \ldots, \alpha_{n}\right), a_{\alpha}=1 / m !^{n} \beta !\left(\partial^{|\beta|} F / \partial x_{10}^{\alpha_{1}} \cdots \partial x_{n 0}^{\alpha_{n}}\right)\left(x^{0}\right)$,

we have $F(x)=\sum_{|\alpha|=0}^{\infty} a_{\alpha}\left(x_{1}-x_{1}^{0}\right)^{\alpha_{1}} \cdots\left(x_{n}-x_{n}^{0}\right)^{\alpha_{n}}$, thus proving that $F$ is $A$-analytic.

$2^{\text {nd }}$ ) The second proof relies on the Taylor formula below which is of interest by itself. Essentially this formula tells us that an $A$-differentiable function of class $C^{r+1}$ can be approximated around a point by a suitable $A$-polynomial of degree $r$ plus a remainder.

Proposition 1. Let $F$ be an A-differentiable function of class $\mathcal{C}^{r+1}$ defined on a neighbourhood $\left|x-x^{0}\right|<\epsilon$ of a point $x^{0} \in A^{n}$. Then we have:

$$
\begin{aligned}
F(x)=\sum_{|\alpha|=0}^{r} \frac{1}{\alpha !} & \frac{\partial^{|\alpha|} F}{\partial x^{\alpha}}\left(x^{0}\right)\left(x-x^{0}\right)^{\alpha} \\
& +\sum_{|\alpha|=r+1} \int_{0}^{1} \frac{(1-t)^{r}}{\alpha !} \frac{\partial^{r+1} F}{\partial x^{\alpha}}\left(x^{0}+t\left(x-x^{0}\right)\right)\left(x-x^{0}\right)^{\alpha} d t
\end{aligned}
$$

Proof of Proposition 1. From Taylor's formula with integral remainder we obtain

$$
\begin{aligned}
F(x)=\sum_{k=0}^{r} & \frac{1}{k !} D^{k} F\left(x^{0}\right)\left(x-x^{0}, \stackrel{k}{k}_{.}, x-x^{0}\right) \\
& +\int_{0}^{1} \frac{(1-t)^{r}}{r !} D^{r+1} F\left(x^{0}+t\left(x-x^{0}\right)\right)\left(x-x^{0}, \stackrel{(r+1,}{\cdots}, x-x^{0}\right) d t,
\end{aligned}
$$

and we can conclude as in the proof of Theorem 2.3 of [4]; i.e., since $F$ is $A$ differentiable we have $D F\left(x^{0}\right)\left(x-x^{0}\right)=\sum_{i}\left(\partial F / \partial x_{i}\right)\left(x^{0}\right)\left(x_{i}-x_{i}^{0}\right), D^{2} F\left(x^{0}\right)(x-$ $\left.x^{0}, x-x^{0}\right)=D(D F)\left(x^{0}\right)\left(x-x^{0}\right)\left(x-x^{0}\right)=\sum_{i, j}\left(\partial^{2} F / \partial x_{i} \partial x_{j}\right)\left(x^{0}\right)\left(x_{i}-x_{i}^{0}\right)\left(x_{j}-\right.$ $\left.x_{j}^{0}\right)$, and so on. 
The proof of the second part of Theorem I is then a simple consequence of the above formula. In fact, we know that if $F$ is real analytic then there exist a neighbourhood $N$ of $x$, and two positive constants $M, \rho$, such that for every $x \in N$, and every $\alpha \in \mathbb{N}^{n}$, we have $\left|(\alpha !)^{-1}\left(\partial^{|\alpha|} F / \partial x^{\alpha}\right)(x)\right| \leq M \rho^{|\alpha|}$, and the result follows by simply bounding the remainder term in Taylor's formula.

\section{Proof of Theorem II and of the Corollary}

Lemma 2. Let $A$ be a finite-dimensional commutative local $\mathbb{R}$-algebra and let $\mathfrak{m}$ be its maximal ideal. If $A / \mathfrak{m} \cong \mathbb{C}$, then $A$ admits a structure of $\mathbb{C}$-algebra compatible with its structure of $\mathbb{R}$-algebra.

Proof. As $\mathfrak{m}$ is nilpotent, it is clear that $A$ is $\mathfrak{m}$-adic complete. From a classical result by I. S. Cohen (see e.g., [1, II.8.25A]) there is a subfield $K \subset A$, containing $\mathbb{R}$, such that $K \rightarrow A / \mathfrak{m}$ is an isomorphism.

Lemma 3. Let $A$ be a finite-dimensional commutative local $\mathbb{R}$-algebra such that $A / \mathfrak{m} \cong \mathbb{R}$. There exist a system of elements $e_{1}, \ldots, e_{r} \in \mathfrak{m}$ and a set of multi-indices $M \subset \mathbb{N}^{r}$ such that:

(i) The cosets of $e^{\alpha}=e_{1}^{\alpha_{1}} \cdots e_{r}^{\alpha_{r}},|\alpha|=l, \alpha \in M$, modulo $\mathfrak{m}^{l+1}$ are a basis of the $\mathbb{R}$-vector space $\mathfrak{m}^{l} / \mathfrak{m}^{l+1}$ for every $l \leq n-1, n$ being the least integer such that $\mathfrak{m}^{n}=0$.

(ii) The elements $e^{\alpha}, \alpha \in M$, with $e^{0}=1$, are a basis of $A$ as a $\mathbb{R}$-vector space.

Proof. Let $e_{1}, \ldots, e_{r}$ be elements of $\mathfrak{m}$ whose cosets are a basis of the $\mathbb{R}$-vector space $\mathfrak{m} / \mathfrak{m}^{2}$. From Nakayama's lemma, $e_{1}, \ldots, e_{r}$ also generate $\mathfrak{m}$ as an $A$ module; that is, every $a \in A$ can be written as $a=a_{1} e_{1}+\ldots+a_{r} e_{r}$ for some $a_{i} \in A$. Assume we have constructed a set of multi-indices $M_{l} \subset \mathbb{N}^{r}$ of order $|\alpha|=l$ so that the cosets of $e^{\alpha}, \alpha \in M_{l}$, are a basis of $\mathfrak{m}^{l} / \mathfrak{m}^{l+1}$. As $e_{1}, \ldots, e_{r}$ generate the $A$-module $\mathfrak{m}$, it is clear that all the elements $e^{\alpha},|\alpha|=l+1$, generate $\mathfrak{m}^{l+1}$. Hence each $a \in \mathfrak{m}^{l+1}$ can be written as $a=\sum_{|\alpha|=l+1} a_{\alpha} e^{\alpha}$ and since $A / \mathfrak{m} \cong \mathbb{R}$, there exist scalars $\lambda_{\alpha} \in \mathbb{R}$ such that $a_{\alpha}-\lambda_{\alpha} \in \mathfrak{m}$. Hence, $a \equiv \sum_{|\alpha|=l+1} \lambda_{\alpha} e^{\alpha}\left(\bmod \mathfrak{m}^{l+2}\right)$. Accordingly, the cosets of $e^{\alpha},|\alpha|=l+1$, generate $\mathfrak{m}^{l+1} / \mathfrak{m}^{l+2}$ as a vector space and from them we can select a basis. In other words, there exist a set of multi-indices $M_{l+1}$ of order $|\alpha|=l+1$ such that the cosets of the elements $e^{\alpha}, \alpha \in M_{l+1}$, are a basis for $\mathfrak{m}^{l+1} / \mathfrak{m}^{l+2}$. Proceeding by recurrence, it will suffice to put $M=\bigcup_{l=0}^{n-1} M_{l}$.

As for (ii), first let us assume we have a relation $\sum_{\alpha \in M} \lambda_{\alpha} e^{\alpha}=0$. Reducing it modulo $\mathfrak{m}$ we obtain $\lambda_{0}=0$. Reducing it modulo $\mathfrak{m}^{2}$ we obtain $\lambda_{1}=\ldots=\lambda_{r}=0$, and so on. Moreover such elements generate $A$ as a vector space, because given $a \in A$ there is an scalar $\lambda_{0}$ such that $a^{\prime}=a-\lambda_{0} \in \mathfrak{m}$. Again $a^{\prime}=a_{1} e_{1}+\ldots+a_{r} e_{r}$ and there are scalars $\lambda_{1}, \ldots, \lambda_{r}$ so that $a_{i}=\lambda_{i}+a_{i}^{\prime}, a_{i}^{\prime} \in \mathfrak{m}$; hence $a=\lambda_{0}+\lambda_{1} e_{1}+\ldots+\lambda_{r} e_{r}+a^{\prime \prime}, a^{\prime \prime} \in \mathfrak{m}^{2}$, and this process finishes after a finite number of steps because $\mathfrak{m}$ is nilpotent. 
Proof of Theorem II. Let $A=A_{1} \times \ldots \times A_{l}$ be the decomposition of $A$ in local algebras. If for every $i$ is $A_{i} / \mathfrak{m}_{i} \cong \mathbb{C}$, then, by virtue of Lemma $2, A$ is a $\mathbb{C}$ algebra and Cauchy's integral formula holds for $\mathbb{C}$-algebras (see [2]); hence the statement is true in that case. In fact, in [2] the author works with algebras of the form $\mathbb{C} \otimes_{\mathbb{R}} B$, where $B$ is a $\mathbb{R}$-algebra, because he assumes the $\mu^{\prime} s$ are real, but his theory also applies to arbitrary $\mathbb{C}$-algebras. Therefore, it will suffice to prove that our statement does not hold if an index $i$ exists at least such that $A_{i} / \mathfrak{m}_{i} \cong \mathbb{R}$. We can assume $i=1$.

It will suffice to construct a function $f: A_{1} \longrightarrow A_{1}$ which is $A_{1}$-differentiable but not $C^{\omega}$-analytic, because prolongating $f$ by zero on the rest of components we will obtain a function $\bar{f}: A \longrightarrow A$ which is $A$-differentiable but not $\mathcal{C}^{\omega}$ analytic and we could conclude by virtue of Theorem I. Consequently, we can assume that $A$ is local and $A / \mathfrak{m} \cong \mathbb{R}$.

Let $f_{0}: \mathbb{R} \longrightarrow \mathbb{R}$ be a $C^{\infty}$ but not $C^{\omega}$ function. Identifying $\mathbb{R}$ with $A / \mathfrak{m}$ and taking into account that $\mathfrak{m}^{n}=0$, it will suffice to construct a $\left(A / \mathfrak{m}^{l}\right)$ differentiable function $f_{l-1}: A / \mathfrak{m}^{l} \longrightarrow A / \mathfrak{m}^{l}$, for every $l=1, \ldots, n-1$, making commutative the diagram below for $l \geq 2$ :

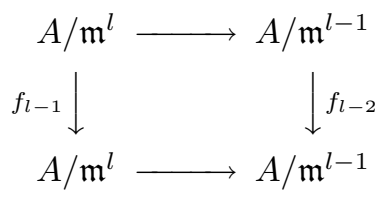

We proceed by recurrence assuming $f_{0}, \ldots, f_{l-1}$ have been constructed and we shall then construct $f_{l}$. To do this we shall use the basis $\left\{e^{\alpha}, \alpha \in M\right\}$ of Lemma 3 and we shall denote by $x_{\alpha}$ the coordinates in this basis; i.e., $a=\sum_{\alpha \in M} x_{\alpha}(a) e^{\alpha}$, so that $A$-differentiability is expressed as $\partial f / \partial x_{\alpha}=$ $\left(\partial f / \partial x_{0}\right) e^{\alpha}, \alpha \in M,|\alpha|>0$. Let us denote by $[a]_{l-1}$ the coset of an element $a \in A$ in $A / \mathfrak{m}^{l}$. We note that $\left\{\left[e^{\alpha}\right]_{l-1} ; \alpha \in M,|\alpha| \leq l-1\right\}$ is a basis for $A / \mathfrak{m}^{l}$. We set $M_{l}=\{\alpha \in M ;|\alpha| \leq l\}$. Thus, $f_{l-1}=\sum_{\alpha \in M_{l-1}} f_{l-1, \alpha}\left[e^{\alpha}\right]_{l-1}$. The commutativity of the above diagram means $f_{l-1, \alpha}=f_{l-2, \alpha}$ for every $\alpha \in M_{l-2}$; hence $f_{l-1, \alpha}$ only depends on the variables $x_{\beta}, \beta \in M_{l-1},|\beta| \leq|\alpha|$. As $f_{l-1}$ is $\left(A / \mathfrak{m}^{l}\right)$-differentiable, for every $|\beta|>0, \beta \in M_{l-1}$, we have

$$
\frac{\partial f_{l-1}}{\partial x_{\beta}}=\sum_{\alpha \in M_{l-1}} \frac{\partial f_{l-1, \alpha}}{\partial x_{\beta}}\left[e^{\alpha}\right]_{l-1}=\sum_{\alpha \in M_{l-1}} \frac{\partial f_{l-1, \alpha}}{\partial x_{0}}\left[e^{\alpha+\beta}\right]_{l-1} .
$$

Hence,

$$
\begin{gathered}
\frac{\partial f_{l-1, \alpha}}{\partial x_{\beta}}=\frac{\partial f_{l-1, \alpha-\beta}}{\partial x_{0}}, \quad \alpha-\beta \in M_{l-1},|\beta|>0, \alpha, \beta \in M_{l-1}, \\
\frac{\partial f_{l-1, \alpha}}{\partial x_{\beta}}=0, \quad \alpha-\beta \notin M_{l-1},|\beta|>0, \alpha, \beta \in M_{l-1} .
\end{gathered}
$$

Let us define $f_{l}$ by giving its components: 


$$
\begin{cases}f_{l, \alpha}=f_{l-1, \alpha} & \text { if } \alpha \in M_{l-1} \\ f_{l, \sigma}=\frac{\partial f_{l-1,0}}{\partial x_{0}} x_{\sigma} & \text { if } \sigma \in M_{l}-M_{l-1}\end{cases}
$$

thus also proving the commutativity of the above diagram for the order $l+1$. Let us check that $f_{l}$ is $\left(A / \mathfrak{m}^{l+1}\right)$-differentiable as well. For every $\gamma \in \mathbb{N}^{r}$ we have:

$$
\begin{aligned}
\frac{\partial f_{l}}{\partial x_{\gamma}}=\sum_{\alpha \in M_{l-1}} \frac{\partial f_{l-1, \alpha}}{\partial x_{\gamma}}\left[e^{\alpha}\right]_{l} \\
\quad+\sum_{\sigma \in M_{l}-M_{l-1}}\left(\frac{\partial^{2} f_{l-1,0}}{\partial x_{\gamma} \partial x_{0}} x_{\sigma}+\frac{\partial f_{l-1,0}}{\partial x_{0}} \delta_{\gamma, \sigma}\right)\left[e^{\sigma}\right]_{l} .
\end{aligned}
$$

Consequently, for $|\gamma|>0, \gamma \in M_{l-1}$, we have $\frac{\partial f_{l}}{\partial x_{\gamma}}=\sum_{\alpha \in M_{l-1}} \frac{\partial f_{l-1, \alpha}}{\partial x_{\gamma}}\left[e^{\alpha}\right]_{l}$. Furthermore,

$$
\frac{\partial f_{l}}{\partial x_{0}}=\sum_{\alpha \in M_{l-1}} \frac{\partial f_{l-1, \alpha}}{\partial x_{0}}\left[e^{\alpha}\right]_{l}+\sum_{\sigma \in M_{l}-M_{l-1}}\left(\frac{\partial^{2} f_{l-1,0}}{\partial x_{0}^{2}} x_{\sigma}\right)\left[e^{\sigma}\right]_{l},
$$

and since $\left[e^{\sigma+\gamma}\right]_{l}=0$ because $|\sigma+\gamma|=|\sigma|+|\gamma|=l+|\gamma| \geq l+1$, we also have

$$
\frac{\partial f_{l}}{\partial x_{0}}\left[e^{\gamma}\right]_{l}=\sum_{\alpha \in M_{l-1}} \frac{\partial f_{l-1, \alpha}}{\partial x_{0}}\left[e^{\alpha+\gamma}\right]_{l} .
$$

By virtue of (3.1) and (3.2) it follows that $\partial f_{l} / \partial x_{\gamma}=\left(\partial f_{l} / \partial x_{0}\right)\left[e^{\gamma}\right]_{l}$. Assume $|\gamma|>0, \gamma \in M_{l}-M_{l-1}$. In that case, (3.4) takes the form $\partial f_{l} / \partial x_{\gamma}=$ $\left(\partial f_{l-1,0} / \partial x_{0}\right)\left[e^{\gamma}\right]_{l}$ because $f_{l-1, \alpha}$ does not depend on $x_{\gamma}$, and from (3.5) we obtain $\left(\partial f_{l} / \partial x_{0}\right)\left[e^{\gamma}\right]_{l}=\sum_{\alpha \in M_{l-1}}\left(\partial f_{l-1, \alpha} / \partial x_{0}\right)\left[e^{\alpha+\gamma}\right]_{l}=\left(\partial f_{l-1,0} / \partial x_{0}\right)\left[e^{\gamma}\right]_{l}$, since for every $|\alpha|>0$ we have $|\alpha+\gamma|=|\alpha|+l \geq l+1$ and $|\sigma+\gamma|=|\sigma|+|\gamma|=2 l \geq l+1$, thus concluding that $\partial f_{l} / \partial x_{\gamma}=\left(\partial f_{l} / \partial x_{0}\right)\left[e^{\gamma}\right]_{l}$ also in this case and thus finishing the proof.

Proof of the Corollary. It follows directly from Theorem II and the following results.

Lemma 4. Let $A$ be a finite-dimensional commutative local $\mathbb{R}$-algebra. $G(A)$ has two connected components if $A / \mathfrak{m} \cong \mathbb{R}$ and it is connected if $A / \mathfrak{m} \cong \mathbb{C}$.

Proof. We have a short exact sequence of Lie groups,

$$
1 \longrightarrow 1+\mathfrak{m} \longrightarrow G(A) \longrightarrow(A / \mathfrak{m})^{*} \longrightarrow 1
$$

Hence $G(A)$ is a principal bundle on $(A / \mathfrak{m})^{*}$ with structure group $1+\mathfrak{m}$ which is homeomorphic to the vector space $\mathfrak{m}$. Consequently, $G(A)$ and $(A / \mathfrak{m})^{*}$ have the same number of connected components. 
Proposition 2. Let $A$ be a finite-dimensional commutative $\mathbb{R}$-algebra, let $A=$ $A_{1} \times \ldots \times A_{l}$ be its decomposition in local algebras and let $\mathfrak{m}_{i}$ be the maximal ideal of $A_{i}$. Then, $G(A)$ is connected if and only if $A_{i} / \mathfrak{m}_{i} \cong \mathbb{C}$ for every $i=1, \ldots, l$. Proof. $G(A) \cong G\left(A_{1}\right) \times \ldots \times G\left(A_{l}\right)$. Hence $G(A)$ is connected if and only if each $G\left(A_{i}\right)$ is, and we can conclude by applying Lemma 4 .

\section{References}

[1] R. Hartshorne, Algebraic Geometry, Springer-Verlag, New York, 1977.

[2] P. W. Ketchum, Analytic functions of hypercomplex variables, Trans. Amer. Math. Soc. 30 (1928), 641-667.

[3] W. C. Waterhouse, Analyzing some generalized analytic functions, Exposition. Math. 10 (1992), 183-192.

[4] W. C. Waterhouse, Differentiable functions on algebras and the equation $\operatorname{grad}(w)=M \operatorname{grad}(v)$, Proc. Roy. Soc. Edinburgh Sect. A 122 (1992), 353361.

P. M. Gadea: Instituto de Matemáticas y Física Fundamental, Consejo Superior de Investigaciones Científicas, Serrano 123, 28006-Madrid, Spain. email: pmgadea@gugu.usal.es

J. Muñoz Masqué: Instituto de Física Aplicada, Consejo Superior de Investigaciones Científicas, Serrano 144, 28006-Madrid, Spain. email: vctqu01@cc.csic.es 